Rev. Elev. Méd. Vét. Poys trop., 1967, 20, 4 (557-562)

\title{
La Distomatose à Madagascar Cycle de Fasciola gigantica
}

\author{
par Pierre DAYNES
}

\begin{abstract}
RÉSUMÉ
L'auteur fait le paint de la Dislomatose chez les ruminants d̀ Madagascar. II décrit le cycle, réalisé expérimentalement, de Fasciola gigantica nouvellement reconnue dans le pays. L'hôte intermédiaire est Lymnaeo natalensis (var. L. hovarum). Les hôtes définitifs utilisés sont le zébu, le mouton, la chèvre.
\end{abstract}

\section{INTRODUCTION}

De l'étude des archives du Service d'Helminthologie (11), il ressort que la présence de Douves semble avoir été signalée pour la première fois à Madagascar en 1904 sur des ovins de la ferme de I'IBOAKA (Province de Fianarantsoa) (5). Mais il est surtout fait mention d'une «cachexie ictéro-vermıneuse » ayant entraîné la mort de « moutons malgaches ».

En 1905 par contre, il est précisé que les foies des moutons morts à la même ferme contiennent des « distomes » sans qu'il soit précisé cependant de quels moutons il s'agit non plus d'ailleurs que de quels distomes.

Puis c'est en 1917 que I'on signale des « Douves» dans les foies de moutons à Antsirabe (Province de Tananarive) soit à la Station d'Antsirabe même, soit chez un colon du voisinage.

Dans les 2 cas précédents, il semble qu'il se soit agi de moutons importés de France.

POISSON (6) parlant des ovins importés (Mérinos d̀ Nanisana-Tananarive en $1896-$ Solognots et Algériens à Nanisana - Tananarive en 1901) et des brebis malgaches saillies estime «très vraisemblable de penser que certains de ces animaux eurent le foie parasité par la « petite douve »... ».

En 1929 on trouve des «Douves» chez des brebis Dishley-Mérinos à la ferme de Vavavato près de Betafo (Antsirabe) et sur des ovins importés de Camargue à la ferme d'Ambovombe (Province de Tuléar, près de Fort-Dauphin). Dans ce dernier cas c'est Fasciola hepotica qui est expressement reconnue.

Ces Fasciola ne se sont pas multipliées et il suffit de rappeler que les Limnées du groupe Lymnoed truncotula sont absentes de Madagascar pour qu'on le comprenne aussitôt.

POISSON ef ADVIER (7) font le rapprochement entre les trématodes en cause chez les ovins et les limnées; ils précisent que ces dernières sont très voisines de Lymnaea natalensis.

Mais en fait, jusque-là, les observations sûres faites ne concernent que les ovins importés.

En ce qui concerne les grands ruminants, diverses importations ont introduit $d u$ bétail d'Europe, d'Amérique, d'Asie et d'Afrique, mais nous ne trouvons signalée nulle part la présence de Fasciola.

Les animaux que nous avons pu examiner à l'occasion d'importations entre 1960 et 1966 provenalent d'Afrique (10 bovins Sahiwal en 1962) et d'Europe (bétail norvégien en 1965 , Ovins d'Arles en 1966). Les coproscopies systématiques ne nous ont pas montré d'œufs de Fasciola sur les uns et sur les autres.

Des animaux Frisons importés de France en 1966 étaient porteurs de $F$. hepotica, ce qui a été reconnu par la coproscopie et confirmé par une autopsie effectuée sur une vache morte pour d'autres raisons.

En 1966, a été signalée pour la première fois 
à Madagascar la présence de F. gigantica (2). Cette douve a été trouvée sur un bovin âgé de 5 ans environ et né d̀ Madagascar. Cet animal, une vache de race «Rana» avait vécu 3 ans environ dans la région de Tananarive puis vivait dans le Moyen-Ouest sur des sols ferrallitiques dans des pâturages composés d'Heteropogon, d'Hyparhenia et d'Aristida et fréquentait des bas fonds tourbeux où dominent les Cypéracées avec Leersia hexandra ef Panicum glanduliferum. Dans ces bas fonds le $\mathrm{pH}$ du sol est de l'ordre de 4,5 et le $\mathrm{pH}$ de l'eau est de l'ordre de 6.

Les coproscopies réalisées sur les autres animaux du troupeau auquel appartenait cet animal ont montré chez un certain nombre d'animaux la présence d'œufs qui nous ont fait penser à Fasciola. Ces ceufs mesurent 160 d̀ 180 microns de long et 90 microns de large et présentent une coloration jaunâtre. Ils ont la forme assez typique d'œufs de Trématodes, en amphore et operculés. Des autopsies pratiquées sur ceux de ces animaux qui sont morts ont confirmé la présence de $F$. gigantica.

Dans les bas-fonds fréquentés par ces animaux nous avons rencontré des Biomphalaria et des Lymnaea. Ces derniers gastéropodes nous ont paru se rapporter à l'espèce Lymnaea hovarum (Tristam) (*). Ces Limnées étudiées se sont montrées infestées de formes larvaires de trématodes divers (Métacercaires d'échinostomes, Sporocystes indéterminés, Xiphidiocercaires, Furcocercaires) ef dans certains cas de Cercaria pigmentosa, forme larvaire de Fasciola gigantica. Dix sept Limnées ont été trouvées porteurs de formes larvaires se rapportant à $F$. gigantica (rédies ou cercaires) avec ou sans formes larvaires appartenant à d'autres trématodes. Nous avons décidé de confirmer ces résultats par une infestation expérimentale de ruminants et de réaliser le cycle expérimental de Fasciola gigantica afin d'éliminer toute erreur.

\section{GÉNÉRALITÉS}

Le cycle des fasciola est un cycle typique de Trématode, avec multiplication asexuée chez

(*) Lymnaea hovarum semble n'être qu'une variété de l'espèce $L$. natalensis elle-même appartenant au groupe L. auricularia (voir plus loin). l'hôfe intermédiarre. Dans le milieu extérieur l'cuf, après incubation, donne un miracidium qui va infester un mollusque gastéropode pulmoné aquatique où il se transforme successivement en sporocystes, rédies, et cercaires; ces dernières quittent le mollusque pour aller, sur un support, s'enkyster en métacercaires qui deviennent vite infestantes pour les ruminants.

Les hôtes intermédicires sont des mollusques gastéropodes pulmonés dulçaquicoles. II s'agit en général de Limnées. Lymnaea truncatula et toutes les limnées de même groupe sont en cause lorsqu'il s'agit de Fasciola hepatica. Lymnaea auriculario ef toutes les espèces du même groupe sont en cause dans le cas de Fasciola gigantica. Pour cette dernière, Physopsis africana (Bulinus africanus) est également signalée cornme hôte intermédiaire par NEVEU LEMAIRE (4) et par EUZEBY (3) mais non par A. PORTER (8) et (9).

\section{MATÉRIEL ET MÉTHODES}

Le but recherché était de fermer le cycle de Fasciola gigantica à l'aide de matériel infestant (œufs de $F$. gigantica) provenant d'un bovin trouvé infesté natureliement et de Limnées neuves obtenues par descendance de Limnées récoltées dans le pays.

Le protocole expérimental a été le suivant :

- Elevage de Limnées sauvages et obtention d'une descendance.

- Récolte d'cufs de Fasciola gigantica.

- Infestation de Limnées neuves.

- Obtention de métacercaires de Cercaria pigmentoso.

- Infestation de ruminants neufs.

- Récalte de F. gigantica sexuellement mûres sur les ruminants infestés.

\section{A) Elevage des limniées.}

Nous avons essayé plusieurs méthodes d'élevage : Pontes de mollusques placés en cristallisoirs d̀ $28^{\circ} \mathrm{C}$ avec sable et eau provenant du même bas fond que les limnées ; Limnées placées dans les mêmes conditions et pondant dans ces cristallisoirs; Eclosion de pontes en boîte de Pétri et transport des jeunes mollusques dans les cristallisoirs ci-dessus. Aucune de ces méthodes n'a donné de bons résultats. 
Des élevages en grands aquariums rectangulaires de 40 à 50 litres ont été couronnés de succès.

Ces aquariums sont en verre. Le fond est tapissé sur 3 à $4 \mathrm{~cm}$ de sable de rivière lavé. L'eau utilisée est celle du robinet mais comme elle contient beaucoup de chlore, nous la laissons reposer au moins 8 jours en flacon ouvert avant de l'utiliser dans les aquariums. Des sagittaires sont plantées dans le sable. L'eau est aérée en permanence grâce d̀ un compresseur et un diffuseur. La température de l'eau se situe autour de $27-28^{\circ} \mathrm{C}$ grâce à une canne chauffante à rhéostaf. Le $\mathrm{pH}$ de l'eau se situe aux environs de 8.

Dans ces aquariums sont introduites des Limnées provenant du bas-fond nous intéressant. Celles-ci sont nourries à l'aide de salade dont nous plaçons quelques morceaux à la surface de l'eau, morceaux remplacés tous les 2 ou 3 jours. Dans ces conditions les Limnées ont vécu plusieurs semaines. Elles ont pondu soit à la surface des feuilles de sagittaires, soit sur les vitres de l'aquarium, puis elles sont mortes. L'eau était changée par $1 / 3$ toutes les semaines par siphonnage. Dans l'aquarium les pontes ont donné des mollusques libres en moins de 2 semaines; certains ont pu se développer et vivre plus de 8 mois dans ce même aquarium.

Ces mollusques neufs ont servi d̀ l'infestation par des miracidiums de Fasciola gigantica.

\section{B) Récoltes d'œufs de F. gigantica.}

Une vache du même troupeau que la première vache à Fasciolose signalée, meurt et son foiese montre très infesté de $F$. gigantica. La bile de la vésicule biliaire apparaît riche en œufs de Douve. Cette bile est récoltée puis diluée dans du sérum physiologique. On laisse ensuite sédimenter puis on décante doucement.

\section{C) Infestation de Limnées neuves.}

Ce sont les Limnées neuves obtenues comme décrit ci-dessus qui sont infestées. A la date de l'infestation, elles sont âgées d'environ un mois et demi.

Le sédiment obtenu à partir de la bile diluée, sédiment riche en œufs de Fasciola, est tout simplement versé dans l'aquarium. II n'a pu être fait de contrôle de l'éclosion des cufs, de l'infestation précise des Limnées par les miracidiums éclos, de même qu'il n'a été fait aucun contrôle de l'évolution de l'infestation chez les Limnées. La date exacte d'infestation des mollusques n'est donc pas connue. On a pu noter seulement une mortalité importante chez les mollusques entre le $60^{\mathrm{e}}$ jour et le $75^{\mathrm{e}}$ jour après que les œufs aient été placés dans l'aquarium.

\section{D) Obtention des métacercaires}

Cent jours après que l'aquarium ait été infesté trois Limnées y sont prises au hasard. Elles sont mises chacune dans un petit cristallisoir avec de l'eau ef placées à la chaleur et à la lumière du soleil sur un rebord de fenêtre. Elles émettent alors, toutes les trois, de nombreuses cercaires correspondant à Cercaria pigmentosa, cercaires qui s'enkystent assez vite sur les parois des cristallisoirs ou sur la coquille du mollusque et très vite sur la lame porte objet si on les $y$ transporte pour les étudier.

Les mollusques sont ouverts et se montrent très infestés de rédies et de cercaires, ces dernières s'enkystent assez vite sur les parois et les morceaux de coquilles.

\section{E) Infestation de ruminants neufs.}

Les métacercaures enkysiées sur les parois des cristallisoirs ayant contenu les Limnées sont « décollées » délıcatement à l'aide d'un pinceau et repoussées au fond du cristallısoir. Les morceaux de coquilles sur lesquels sont fixées beaucoup de métacercaires sont également conservés au fond du cristallisoir.

Tenant compte d'une remarque de TAYLOR (10), nous avons laissé «mûrir» ces métacercaires avant d'infester des ruminants. Afin de conserver une certaine humidité, les petits cristallisoirs ont été placés sur un coussin de coton très humide dans une boîte de verre fermée. Cette mâturation permet à la petite douve enfermée dans son kyste d'acquérir toute sa maturité ef toute sa capacité d'infestation. Après 9 jours de maturation les métacercaires sont administrées à un bovin «B 1 » ef à un ovin «O1» dans un bol alimentaire. Celui-ci est constitué de pâte de farine et d'eau sur laquelle se collent facilement les métacercaires. 
Le bovin reçoit une centaine de métacercaires. L'ovin reçoit une cinquantanne de métacercaires.

\section{F) Récolte de F. gigantica chez les riminants infestés.}

Chez le bovin «B 1 » l'éosinophilie passe de 4 p. 100 d̀ 7 p. 100 en trois mois puis atteint un maximum de 12,5 p. 100 cinq mois après l'infestation. Chez l'ovin «O 1 » l'éosinophilie passe de 3 p. 100 d̀ 22 p. 100 en trois mols pour atteindre 30,5 p. 100 cinq mois après l'infestation.

Ces 2 animaux sont contrôlés du point de vue élimination des œufs de douves par des coproscopies bi-hebdomadaires d'abord puis effectués tous les 2 jours à partir de deux mois après l'infestation.

Des cufs de Fasciola apparaissent dans les excréments 146 jours après l'infestation pour «B 1 » ef 137 jours après l'infestation pour «O1».

L'ovin est abattu 170 jours après son infestation. Son foie sclérosé crisse sous le couteau. On y note des lésions nettes d'Angiocholite. On récolte 32 douves adultes.

Le bovin est abattu 200 jours après son infestation. Le foie sclérosé porte des traces d'Angiocholite. II contient 41 douves adultes.

\section{G) Infestations parallèles.}

Parallèlement ont été infestés expérimentalement trois autres ruminants, un bovin «B $2 »$, un ovin «O2》 et un caprin «C1》. Ont été employées des métacercaires obtenues à partir de Limnées infestées naturellement et récoltées dans le bas-fond intéressé.

Les métacercaires obtenues comme précédemment et recueillies de la même façon n'ont été gardées pour maturation que 7 jours au lieu de 9.

Le bovin «B 2 » reçoit un grand nombre de métacercaires (plusieurs centaines). Son éosinophilie passe de 3,5 p. 100 à 35 p. 100 en trols mois et atteint après quatre mois un maximum de 42 p. 100. On trouve des œufs de Fasciolo dans ses excréments 111 jours après son infestation. A partir de 6 mois après son infestation il commence à maigrir. Il est abattu 7 mois après son infestation et son foie très sclérosé contient 538 douves adultes. L'ovin « $\mathrm{O} 2$ » reçoit environ une centaine de métacercaires. Son éosinophilie passe de 3 p. 100 à 13 p. 100 en trois mois et atteint un maximum de 19 p. 100 quatre mois après l'infestation. On trouve des ceufs de Fasciola dans ses excréments 123 jours après. son infestation. Abattu 7 mois et demi après l'infestation, il présente une angiocholite avec ectasie des canaux sclérosés. Le foie contient au total 21 douves adultes.

Le caprin «C1》 reçoit une centaine de métacercaires. Son éosinophilie évolue de 3,5 p. 100 d̀ 10 p. i100 en trois mols. Elle atteint 23 p. 100 quinze jours plus tard ef l'animal meurt sans qu'on ait trouvé d'cufs de Foscolia dans ses excréments. Le foie, mou, montre la présence de 60 jeunes douves (de $2 \mathrm{~cm}$ de long environ) présence s'accompagnant d'une forte hépato-mégalie avec congestion et périsplénite.

\section{CONCLUSIONS ET DISCUSSIONS}

Nos expériences nous ont permis de fermer à Madagascar le cycle de Fasciola gigantica.

L'hôte intermédiaire en cause est la Limnée connue sous le nom de Lymnaea hovarum (Tristram). Les mollusques étudiés par RANSON de 1955 d̀ 1960 pour BRYGOO (1) ont été identifiés comme Lymnaea hovarum (Tristam). Selon RAN$S O N$, d'après BRYGOO (1), L. hovarum était probablement une espèce différente de $L$. natolensis KRAUSS.

Le professeur MANDAHL-BARTH (*) a bien voulu étudier notre matériel animal et selon lui, il s'agirait d'une variété de l'espèce $L$. natalensis, répandue en Afrique du Sud. Cette dernière est à rattacher à la Super espèce $L$. auricularia. Ce groupe, qui s'oppose en gros au groupe de L. truncatula, se caractérise, entre autre, biologiquement, par son aptitude à assurer le développement des formes lárvaires de Fasciola gigantica. Le Professeur MANDAHL-BARTH (in lelferis) estime que si L. natalensis peut être considérée comme une espèce du groupe «auricularia» L. hovarum ne peut être considérée comme une sous-espèce de $L$. natalensis mais uniquement comme une variété écologique de cette dernière.

(*) Nous tenons à remercier ici le Professeur MANDAHL-BARTH pour les renseignements qu'il a bien voulu nous fournir. 
C'est ce qu'avaient pressenti POISSON ADVIER (7) sans toutefois le prouver.

Le problème n'ayant pas été approfondi ef aucune expérimentation n'étant réalisée il était tacitement convenu que Madagascar était d'une part indemne de douve, d'autre part à l'abri d'une infestation par les douves*. On se basait sur le fait que les douves introduites n'avaient jamais donné de distomatose sur le bétail malgache et sur l'absence reconnue de Lymnaea truncatula. Or les douves jusqu'ici introduites étaient des Fasciola hepatica et nous-même avons considéré comme non dangereux l'introduction de cet helminthe dans l'lle.

La découverte de F. gigantica à Madagascar conduit à modifier cette opinion systématique sur la « douve ».

Le fait que F. gigantica ait été trouvée sur un bovin né à Madagascar disait assez que le cycle s'était fermé sur place. Restait à la rigueur une quelconque et peu probable possibilité d'introduction de métacercaire avec un fourrage ou une litière indéterminés. Même en admettant cette possibilité il est maintenant prouvé que le cycle de F. gigantica peut se fermer sur place

(*) Le terme Douve signifie ici Fasciola et dicrocoefium. (ce qui était supposable puisqu'on connaissait l'existence de Lymnaea hovarum (Tristram) $(=L$. notalensis).

Il faut noter dans nos résultats, les différences, apparentes tout au moins, d'évolution chez les hôtes définitifs expérimentalement infestés. Les animaux infestés avec des métacercaires obtenues sur des mollusques neufs élevés et infestés en laboratoire ont extériorisé leur infestation avec certitude (Coproscopie) plus tard que les animaux infestés avec des métacercaires provenant de mollusques naturellement infestés dans la nature.

Il faut noter également que le degré de l'éosinophilie qui apparaît lors de l'évolution chez I'hôte définitif semble en rapport ayec le degré de l'infestation.

Enfin, il convient de relever la facilité de développement des métacercaires en douves chez les ruminants infestés : 20 à 60 p. 100 des métacercaires absorbées donnent des douves dans le foie.

Institut d'Flevage et de Médecine Vétérinare des Pays Tropicaux Laboratoire Centrol de l'Elevage Tananarive

\section{SUMMARY}

Liyer-fluke in Madagascar. Biological cycle of Fasciolo gigantico

The problem of the liver-fluke in ruminants in Madagascar has been reviewed. The biological cycle of Fasciola gigantica, which was recently reporled in the Country, has been described and experimentally carried out. The intermediate host is Lymnaea notalensis (var. L. havarum). The permanent hosts used for experiment are the Zebu, the Sheep and the Goat.

\section{RESUMEN}

La distomatosis en Madagascar. Ciclo de Fasciola gigantica

El autor pasa en revista los datos concernientes a la distomatosis de los rumiantes en Madagascar. Describe el ciclo, realizado esperimentalmente, de Fasciola gigantica nuevamente reconocida en el pais. El huésped intermediario es Lymnaea natalensis (var. L. hovarum). Los huéspedes definitivos utılizados son el cebú, la oveja, la cabra. 


\section{BIBLIOGRAPHIE}

1. BRYGOO (E. R.). -- Les Bilharzioses humaines à Madagascar. Arch. Inst. Pasteur Madogoscar, 1965, $33: 144-45$.

2. DAYNES (P.). - Note préliminaire sur la présence de Fasciola gigantica à Madagascar. Rev. Elev. Méd. vét. Pays trop., 1966, 19 (3) : 275-76.

3. EUZEBY (J.). - Les zoonoses helminthiques. Paris, Vigot Frères, 1964.

4. NEVEU-LEMAIRE (M.). - Traité d'helminthologie. Paris, Vigot Frères, 1936.

5. POISSON (H.). - Note sur la distomatose des moutons à Madagascar. Bull. Soc. Poth. exo., 1929, 22 (6) : 521-23.

6. POISSON $\left(H_{.}\right)$, — Nołe sur les lésions du fole des moutons à Madagascar. Bull. Soc. Poth. exo., 1929, 22 (6) : 525-26.
7. POISSON (H.), et ADVIER $\left(M_{1}\right)$. - Note sur une limnée de Madagascar ef sur les cercairés qu'elle héberge. Buil. Soc. Path. exo., 1929,22 (6) : 523-25.

8. PORTER (A.) , - The IIfe history of the African Sheep and Cattle fluke, Fasciola gigantica. S. Afr. J. Sci., 1920-21, 17 : 126-30.

9. PORTER (A.). - The larval trematoda found in certain South Africa mollusca. Publication of the South African Institute for Medical Research, Johannesburg, 1938, 8 (42).

10. TAYLOR (E. L.). - La Fasciolose ef la Douve du Foie. Rome, Organisation des Nations Unies pour l'Alimentation ef I'Agriculture, 1963. (Etude agricole de la F. A.O. No 64).

11. Archives du Service d'Helminthologie collationnées par POISSON $(\mathrm{H}$.). 